\begin{tabular}{|c|l|}
\hline Title & Flow-network adaptation in Phy sarum amoebae \\
\hline Author(s) & Tero, A tsushi; Y umiki, Kenji; Kobay ashi, Ryo; Saigusa, Tetsu; Nakagaki, Toshiyuki \\
\hline Citation & $\begin{array}{l}\text { Theory in Biosciences, 127(2), 89-94 } \\
\text { https://doi.org/10.1007/312064 008-0037-9 }\end{array}$ \\
\hline Issue Date & 2008-06 \\
\hline Doc URL & http://hdl.handle.net/2115/38533 \\
\hline Rights & The original publication is available at www.springerlink.com \\
\hline Type & article (author version) \\
\hline File Information & TheoBiosci20080130.pdf \\
\hline
\end{tabular}

Instructions for use 


\title{
Flow-network adaptation in Physarum amoebae
}

\author{
Atsushi Tero ${ }^{1}$, Kenji Yumiki², Ryo Kobayashi ${ }^{2}$, \\ Tetsu Saigusa $^{3}$, Toshiyuki Nakagaki ${ }^{1,4}$ \\ ${ }^{1}$ Research Institute for Electronic Science, \\ Hokkaido University, Sapporo, 060-0812, Japan \\ ${ }^{2}$ Department of Mathematics and Life Sciences, \\ Hiroshima University, Higashi-Hiroshima 739-8626, Japan \\ ${ }^{3}$ School of Engineering, Hokkaido University, \\ Sapporo 060-8628, Japan \\ ${ }^{4}$ Creative Research Initiative SOUSEI, \\ Hokkaido University, Sapporo, 001-0821, Japan
}

\begin{abstract}
Understanding how biological systems solve problems could aid the design of novel computational methods. Information processing in unicellular eukaryotes is of particular interest, as these organisms have survived for more than a billion years using a simple system. The large amoeboid plasmodium of Physarum is able to solve a maze and to connect multiple food locations via a smart network. The current study examined how Physarum amoebae compute these solutions. The mechanism involves the adaptation of the tubular body, which appears to be similar to a network, based on cell dynamics. Our model describes how the network of tubes expands and contracts depending on the flux of protoplasmic streaming, and reproduces experimental observations of the behavior of the organism. The proposed algorithm based on Physarum is both simple and powerful.

Keywords: cell dynamics, network, Steiner minimum tree, Physarum

Abbreviations: FS, food source; FT, fault tolerance; SMT, Steiner minimum tree; TL, total length of the tube network

\section{Introduction}

Technologies capable of connecting multiple locations, such as public transportation networks between cities, are fundamental to modern society. In general, such networks must have several properties including a minimal total length, tolerance of global connectivity to accidental disconnection of the edges, and a short distance for dense communication. However, it is difficult to meet all of these requirements without some trade-offs. The most popular method for solving such problems is
\end{abstract}


the combinatorial optimization technique, but this approach is time consuming. Two biologically-inspired methods have been proposed to overcome this difficulty: genetic algorithms and ant algorithms [1].

Recently, we reported that the true slime mold Physarum could effectively solve a network problem in just a few days $[2,3]$. Here we propose a mathematical model for connecting multiple locations, and consider how this primitive organism solves such problems.

The Physarum polycephalum plasmodium is a large amoeboid organism with an intracellular structure consisting of a network of tubes. The network acts both as an information highway transporting chemical and physical signals, and as a supply network circulating nutrients and metabolites throughout the organism $[4,5]$. It is also regarded as an organ of movement, as protoplasmic body mass is transported through the tubes. This multi-functional system is therefore of great physiological importance to the plasmodium. Moreover, the computing ability of Physarum is much greater than was previously thought.

The shape of the network alters drastically in response to environmental changes and external stimulation. For instance, when many separate food pellets are presented on an agar plate, a tube network forms to connect them over a short distance. Maze solving is one experimental outcome of this behavior $[6,7,8]$. The organism spreads throughout the available space in a maze when two food sources (FSs) are presented at two exits. The shortest tube path connecting the two FSs is then chosen from the many possible options. Previously, we proposed a mathematical model for the assembly and disassembly of the tube network during maze solving $[9,10]$. Simulations were carried out, and the model was demonstrated to be useful. The resulting method based on Physarum was named Physarum solver [9]. It has been mathematically proven that Physarum solver can find the shortest path in a general planar graph $[11,12]$.

The abovementioned model describes the dynamics of a tube network that balances two antagonistic processes: tube thickening depending on flux through the tube itself, and tube thinning with first-order kinetics. The dynamics can thus be calculated once the flow through every tube is known. The physiological basis for the model has been comprehensively discussed in previous papers $[10,13]$. The behavior with two FSs has been modeled in detail, as it is relatively easy to determine the flow through the tubes in such a case [10].

So far, however, no mathematical model for three FSs has been proposed. This is because, with more than two FSs, it is necessary to know how much sol flows from each individual FS to the others, as the flow of protoplasm through every tube is needed to compute the tube dynamics. Unfortunately, limited data have so far been obtained. Here we apply the tube dynamics to the case of multiple FSs by making some logical assumptions about sol flow. This highlights the importance of the total flux of sol integrated over the entire organism, and of a parameter that expresses the dependence of tube thickening on sol flux. The effects of these parameters are thus examined.

The model reproduces a wide variety of the network shapes observed in real experiments with three FSs. The required properties of the functional network mentioned above are similar in the simulation and the actual organism. The network shapes are shown with four FSs, six FSs, and so on, and we discuss an algorithm derived from Physarum. 


\section{Networking three or more FSs}

\subsection{Mathematical model for a tube network adaptive to flow}

A mathematical model for cases with two FSs has previously been proposed [10]. The model consists of sets of equations for protoplasm flow and adaptability of tube thickness, respectively. The model was based on experimentally observed biological behavior, but took a form that was mathematically simplified and tractable.

In brief, the model represents the shape of the cell body by a graph, in which an edge corresponds to a plasmodial tube and a node corresponds to a junction between tubes. The two nodes with food sources are labeled $N_{1}$ and $N_{2}$ while the other nodes are numbered $N_{3}, N_{4}, N_{5}, \cdots$ and so on. The edge between node $i$ and $j$ is labeled $M_{i j}$, and multiple edges between these nodes are labeled $M_{i j}^{1}, M_{i j}^{2}, \cdots$.

Suppose that the pressures at nodes $i$ and $j$ are $p_{i}$ and $p_{j}$, respectively, and that the two nodes are connected by a cylinder of length $L_{i j}$ and radius $r_{i j}$. Assuming Poiseuille flow, the flux through the tube is a measure of the conductivity of the tube:

$$
Q_{i j}=\frac{\pi r^{4}\left(p_{i}-p_{j}\right)}{8 \xi L_{i j}}=\frac{D_{i j}}{L_{i j}}\left(p_{i}-p_{j}\right),
$$

where $\xi$ is the viscosity of the fluid, and

$$
D_{i j}=\frac{\pi r^{4}}{8 \xi}
$$

Although the tube walls are not rigid and the radius changes over time, the dynamics of tube adaptation are sufficiently slow (10-20 min) for the flow to be considered at steady state. The state of the network is described by the fluxes $Q_{i j}$, and the conductivities, $D_{i j}$, of the edges.

At each node $i(i \neq 1,2)$, the inflow and outflow must be balanced as

$$
\Sigma_{j} Q_{i j}=0 .
$$

The nodes that correspond to food sources drive the flow through the network, so that, at the food sources, eq.3 is modified by a prescribed source (or sink) term. For the source node $(i=1)$ and the sink node $(i=2)$,

$$
\left\{\begin{array}{c}
\Sigma_{j} Q_{1 j}-I_{0}=0, \\
\Sigma_{j} Q_{2 j}+I_{0}=0,
\end{array}\right.
$$

where $I_{0}$ is the flux flowing into the source node and out of the sink node. These source terms might be periodic in time, and drive shuttle streaming through the network. However, because the time scale of network adaptation is an order of magnitude larger than the time scale of shuttle streaming, the sources are taken to be constant. It should be noted that $I_{0}$ is a parameter in our model.

For a given set of conductivities and source and sink, the flux through each of the network edges can be computed. In Physarum, the radii of the tubes change in response to this flux $[10,14]$, and in the model the conductivities evolve according to the equation

$$
\frac{d D_{i j}}{d t}=f\left(\left|Q_{i j}\right|\right)-a D_{i j} .
$$


Here, the first term on the right-hand side describes the expansion of tubes in response to the flux. The function $f$ is a monotonically increasing function that satisfies the condition $f(0)=0$. The second term represents a constant rate of tube constriction and $a$ is a positive constant for the first order kinetics, so that the tubes will disappear in the absence of flow. The tubes interact with one another because the total amount of fluid in the network must be conserved. If the flux through a tube changes, it affects all the other tubes in the network.

It is instructive to consider an analogy with an electrical circuit. An edge of the network is regarded as a dynamic resistor, with resistance proportional to $L_{i j}$ and $r_{i j}^{-4}$. The shape of the organism is represented as a network of resistors. The fluxes through the edges are thus analogous to the currents through the resistors, and the source/sink terms at the food sources correspond to the input currents. The pressures at the nodes correspond to the voltages in the circuit. If the current through a resistor is large enough, its resistance decreases and the current through it increases. If the current through a resistor is low, the resistance increases and finally tends to infinity, which corresponds to the collapse of the tube.

The model used eq. (1)-(5) corresponds to Physarum solver if $f(|Q|)=|Q|^{\mu}$, $(\mu=1)$. However, here Physarum solver is modified to be applicable to cases of more than three FSs. The function form $f(|Q|)$ is given by a more realistic form $f(|Q|)=|Q|^{\gamma} /\left(1+|Q|^{\gamma}\right)$ than the power function. The observation that there is a maximum tube diameter clearly requires the growth function to saturate, regardless of the mechanism. We assume that the flux is proportional to the pressure; however, the thixotropy (which is a property of non-Newtonian fluids) of protoplasm means that it barely flows when the pressure difference is small. Modeling the complex rheology of the protoplasm is beyond the scope of this paper. To account for this observation in our model, we take $\gamma>1$ in the sigmoidal function, so that tube growth is less sensitive to changes in the flow when the pressure differences are small.

In order to calculate the numerical simulation for the modified model, it is necessary to know which FSs are sources of protoplasmic flow and which are sinks. However, this is largely unclear. We thus assume that only one source and one sink exist at any given moment, and that they are not fixed but rather switch at random between all of the FSs. At each time step, one FS node is randomly picked as a sink, say it is node $i$. Then another FS node $j$ is randomly picked as a source with the probability $d_{i j}^{\beta} / \Sigma_{k \neq i} d_{i k}^{\beta}$, where the index $k$ runs over a set of FS nodes and $\beta$ is a positive constant. The $d_{i k}$ is distance between the sink node $i$ and the source node $k$. This probability means that an FS farthest from the sink is most likely to become the source. We have studied the parameters $\gamma, I_{0}, \beta$ of this amended model.

\section{Simulation results and comparison with real networks}

Figure 1 shows the results of a simulation with three FSs. The model succeeds in reproducing a wide variety of network patterns observed in the real organisms. The differences between the patterns result from differences in the parameters. Here, $I_{0}$ is the total flow through the entire network, $\gamma$ is the exponent of a sigmoidal function 
in the process of tube thickening, and $\beta$ is $\beta$ th order of moment for choosing a source node stochastically. In general, the number of edges increases as $I_{0}$ increases at a fixed $\beta(=1)$. The dependence of $I_{0}$ agrees with the experimental observation that the tube network tends to be dense and complex when the organism initially applied is large (so that the flux of protoplasm between the FSs is large).

In our experiments [3], we observed a network shape resembling the so-called Steiner minimum tree (SMT), which is the shortest connection network between multiple locations on a plane (Fig. 1a1). This is not, in fact, an SMT because the edges are not straight, and the junction of the three edges around the centers of the three FS positions is not at the center of mass of the three points. However, the connectivity of nodes and edges is the same as that of an SMT. Other types of network shape were observed, as shown in Fig. 1a. The total length of network increases from Figures 1a1 to 1a5. We noted a statistical tendency for network shapes of greater total length to emerge, as this experimental parameter was increased (that is, the organism was in relative excess over the volume of food provided). This implies that a large amount of protoplasmic sol can be exchanged between FSs.

We observed a similar tendency in the simulation. As the flow rate $I_{0}$ increased from 1.5 to 6.0 (Figs. $1 \mathrm{~b} 1$ and $1 \mathrm{~b} 5$, respectively), the network shape became more complicated, with a longer total length. The model thus reproduced an important behavior of the organism. The other parameter, $\gamma$, was set between 2.5 and 4.0, so that the simulation results might fit the experimental results. This meant that there was no rigorous reason for the choice of parameter value, but similar values were used for the cases of two FSs in our previous paper [15] in order to reproduce the actual behavior of the organism. The issue of parameter choice needs to be studied in future, based on indications from physiological experiments [13]. $\beta$ was set at 1.0 in the abovementioned simulation.

Fig.2 shows the actual network shapes and the simulation results with four FSs, which were located at the vertices of squares and rectangles. Two typical shapes observed in the square setup were the SMT-type and the cycle type (Figs. 2a1 and $2 \mathrm{a} 2$, respectively). The effect of $I_{0}$ was similar to that in the case of three FSs, as the total length was greater in Fig. $2 \mathrm{~b} 2\left(I_{0}=6.5\right)$ than in Fig. $2 \mathrm{~b} 1\left(I_{0}=2.0\right)$. The parameter $\beta$ was $\beta=5.0$ in the SMT-type and only $\beta=3.0$ in the cycle type. In the simulation of the rectangular setup, the SMT type appeared at large values of $\beta$ as shown in Fig. 2b3, while the cycle type was obtained at relatively small values of $\beta$. In general, the SMT type tended to appear at large $\beta$ values in some other sets of FS positions. This implies that the plasmodium might construct an SMT when protoplasmic sol (and chemical and physical signals transported by the sol) is mainly exchanged between maximally separated parts of the organism. This matter also needs to be clarified in future studies.

In the biological experiments with three FSs, the functionality of networking was evaluated by two kinds of measure: shortness of total length of the entire network, and fault tolerance (FT) of the global connectivity to accidental disconnection of the tubes $[2,3]$.

Two measures were used to evaluate the network pattern: the total length of the tube network (TL), and the FT against accidental disconnection of the tubes [2]. TL is expressed as a dimensionless value, which is the total length divided by that of the SMT. $\mathrm{FT}_{N}$ is defined as the probability that the organism (that is, main part of the body located at the food source) is not fragmented if $\mathrm{N}$ accidental 
disconnections occur at random points along the tubes. The $\mathrm{FT}_{1}$ is calculated by assuming that one disconnection occurs, and that the probability of a disconnection is proportional to the ratio of tube length to total network length. For the $\mathrm{FT}_{2}$, two disconnections are assumed. A longer tube has a higher risk of disconnection; therefore, smaller values of TL and higher values of FT are advantageous. These trends result from the physiological requirements of the organism. Generally, FT rises as TL increases, which reflects the relative cost of redundant connections to food sources. Therefore, we tested the FT-TL relationship, which expresses the cost-benefit trade-off, as shown in Figure 3.

Figure 3 shows a comparison of the functionality in the real organism and the simulation results evaluated by the abovementioned measures TL varies widely, as a considerable range of network shapes was observed. For all of the TL values, the FT observed represented the very good value under the constraints of that specific TL (Figs. 3a and 3c). This shows that the organism consistently maximized FT at the different TLs. Figures $3 \mathrm{~b}$ and $3 \mathrm{c}$ show the TL-FT relationship for network shape obtained by the model simulation (Fig. 1b). There is good agreement between the real organism and the simulation. Thus, the model effectively reproduces network formation in the organism.

\section{Conclusion}

We analyzed networking in the biological system of Physarum, and the observed phenomena were reproduced by a mathematical model. Based on the behavior of the organism, we developed a method for computing the networking. The problem of functional networking might thus be solved by computation. In future, we might also learn more from this amoeboid organism, which is capable of finding the shortest connection path between multiple food sources. With a specific set of parameters, the mathematical model reproduces the Steiner minimum tree. Building upon this model behavior, it might be possible to generate an algorithm for a Steiner problem solver. This topic will be considered elsewhere in the near future

\section{Acknowledgments}

This research was supported by Grants-in-aid for Scientific Research (nos. 18650054, 18654022 and 19340023) from the Japan Society for the Promotion of Science and by a Research Grant from the Human Frontier Science Program (no. RGP51/2007).

\section{References}

[1] Dorigo, M., Stützle, T., 2004. Ant Colony Optimization. MIT Press, Massachusetts.

[2] Nakagaki, T., Yamada, H., Hara, M., 2004. Smart network solutions in an amoeboid organism. Biophys. Chem. 107, 1-5.

[3] Nakagaki, T., Kobayashi, R., Nishiura, Y., Ueda, T., 2004. Obtaining multiple separate food sources: Behavioural intelligence in the Physarum plasmodium, Proc. R. Soc. Lond. B 271, 2305-2310. 
[4] Kamiya, N., 1959. Protoplasmic Streaming. Protoplasmatologia 8. Springer, Lewis Victor Heilbrunn.

[5] Kessler, D., 1982. Plasmodial structure and motility, in: Aldrich, H. C., Daniel, J. W. (Eds), Cell biology of Physarum and Didymium Academic Press, New York, pp. 145-196.

[6] Nakagaki, T., Yamada, H., Tóth, A, 2000. Maze-solving by an amoeboid organism Nature 407, 470.

[7] Nakagaki, T., Yamada, H., Tóth, A, 2001. Path finding by tube morphogenesis in an amoeboid organism Biophys. Chem. 92, 47-52.

[8] Nakagaki, T., 2001. Smart behavior of true slime mold in labyrinth. Res. Microbiol. 152, 767-770.

[9] Tero, A., Kobayashi, R., Nakagaki, T., 2006. Physarum solver a biologically inspired method for road-network navigation. Physica A363, 115-119.

[10] Tero, A., Kobayashi, R., Nakagaki, T., 2007. Mathematical model for adaptive transport network in path finding by true slime mold. J. Theor. Biol. 244, 553-564.

[11] Miyaji, T., Onishi, I., 2008. Physarum can solve the shortest path decision problem mathematically rigorously, International Journal of Pure and Applied Mathematics, to appear.

[12] Miyaji, T., Onishi, I., 2007. Mathematical analysis to an adaptive network of the plasmodium system. Hokkaido Mathematical Journal 36, 445-465.

[13] Nakagaki, T., Guy, R., 2008. Intelligent behaviors based on complex dynamics of soft matter in the Physarum plasmodium, Soft Matter 4, 57-67.

[14] Nakagaki, T., Yamada, H., Ueda, T., 2000. Interaction between cell shape and contraction pattern. Biophys. Chem. 84, 195-204.

[15] Nakagaki, T., Saigusa, T., Tero, A., Kobayashi, R., 2007. Effects of amount of food on path selection in the transport network of an amoeboid organism. In: Proc. Int. Symp. On Topological Aspects of Critical Systems and Networks World Scientific Publishing Co., pp. 94-100. 


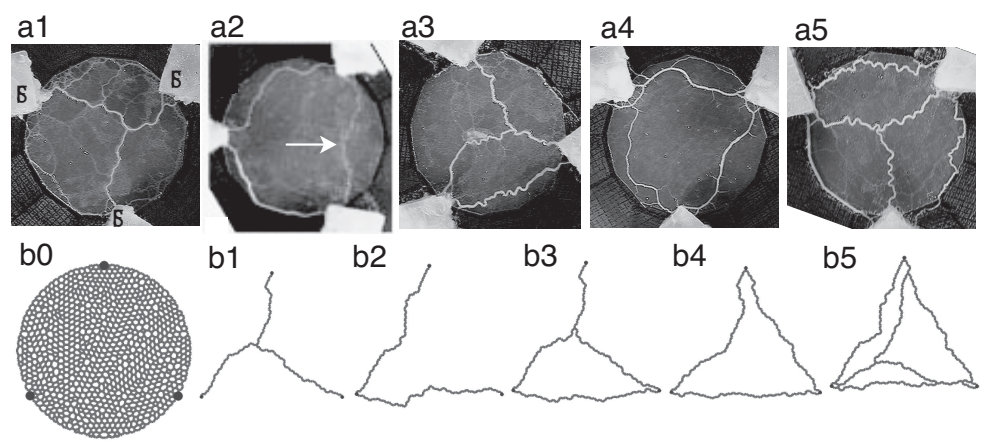

Figure 1: Simulation for three FSs located at the three vertices of an equilateral triangle. (a) Network shapes in the real organism, in ascending order of total length of network, from a1 to a5. (b) Simulated network shapes. (b0) Initial conditions with constant thickness $D_{i j}=1.0$. The parameter sets of $\left(I_{0}, \gamma, \beta\right)$ were $(1.5,3.5,1.0),(3.0,4.0,1.0)$, $(5.0,3.5,1.0),(6.0,3.0,1.0)$, and $(6.0,2.5,1.0)$, for b1, b2, b3, b4, and b5, respectively. $a=1.0$. A wide variety of network shapes were observed in both systems. (a2) The right side edge indicated by the arrow appears to be present, but, in fact, has already disconnected and is actually only a trace of the viscous sheath of the slime mold. 

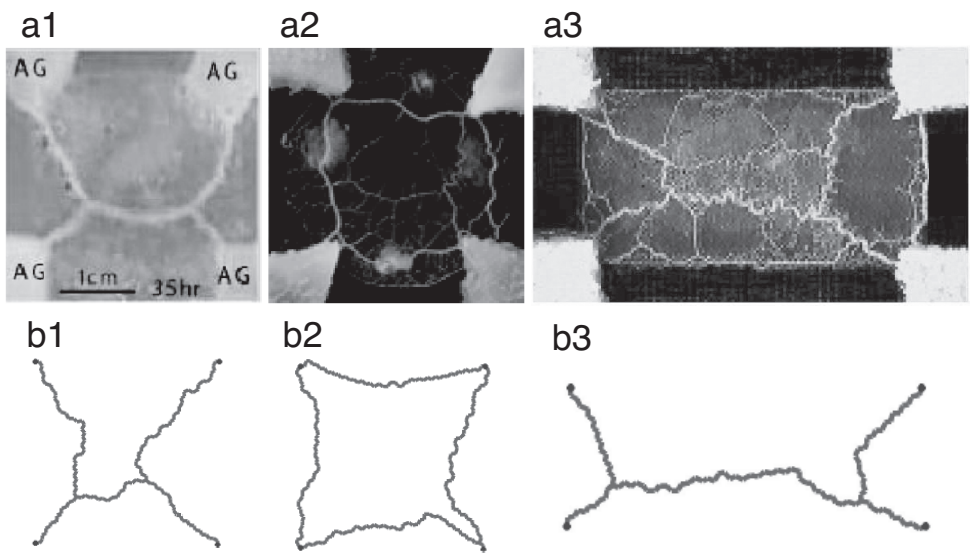

b2

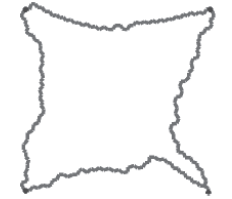

b3

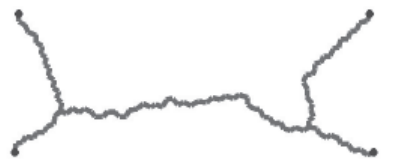

Figure 2: Simulation for four FSs. (a) Real organism. (b) Model simulation. $\left(I_{0}, \gamma\right.$, $\beta)=(2.0,3.5,5.0),(6.5,3.5,3.0)$ and $(2.0,3.5,5.0)$ for $\mathrm{b} 1, \mathrm{~b} 2$, and $\mathrm{b} 3$. Note that $I_{0}$ is the total flow through the entire network, $\gamma$ is the exponent of the sigmoidal function in the process of tube thickening, and $\beta$ is the $\beta$ th order of moment for choosing a source node stochastically $a=1.0$. AG: food-source. 


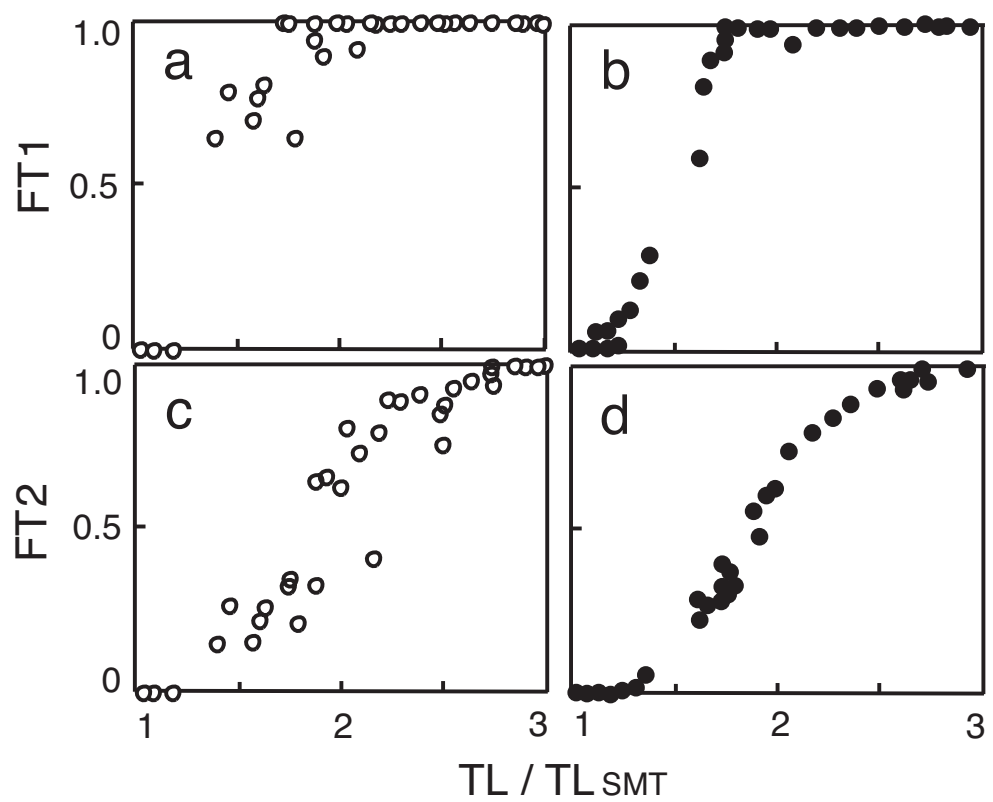

Figure 3: FT of the network in the real organism $(\mathrm{a}, \mathrm{c})$ and in the model $(\mathrm{b}, \mathrm{d})$. (a, b) FT against one accidental disconnection $\left(\mathrm{FT}_{1}\right)$. (c, d) FT against two accidental disconnections $\left(\mathrm{FT}_{2}\right)$. Parameters $I_{0}$ and $\gamma$ were randomly set at any values in the range $3.0<I_{0}<8.0$ and $2.0<\gamma<4.0$, respectively. $\beta=1.0, a=1.0$. 